\title{
FOUR-DISCORDANT PERMUTATIONS
}

\author{
EARL GLEN WHITEHEAD, JR.
}

(Received 14 April 1978; revised 30 May 1979)

Communicated by W. D. Wallis

\begin{abstract}
A permutation $\pi$ of the set $\{1,2, \ldots, n\}$ is four-discordant if $\pi(i) \neq i, i+1, i+2, i+3(\bmod n)$ for $1 \leqslant i \leqslant n$. Generating functions for rook polynomials associated with four-discordant permutations are derived. Hit polynomials associated with four-discordant permutations are studied. Finally, it is shown that the leading coefficients of these rook polynomials form a "tribonacci" sequence which is a generalized Fibonacci sequence.
\end{abstract}

1980 Mathematics subject classification (Amer. Math. Soc.): primary 05 A 05 ; secondary 05 A 15.

\section{Introduction}

The problem considered in this paper is the fourth problem in a hierarchy of problems. These problems involve permutations $\pi_{i}$ defined on the $n$-set $N=\{1,2, \ldots, n\}$ where $\pi_{i}(j) \equiv i+j-1(\bmod n)$, such that $\pi_{i}(j) \in N$.

The first problem in this hierarchy is the probleme des rencontres, also known as the problem of derangements. For a discussion of derangements, see Berman and Fryer (1972). This problem is to enumerate the permutations of an $n$-set discordant with the identity permutation $\pi_{1}$. A permutation $\pi$ is discordant with a given permutation $\pi_{i}$ if $\pi(j) \neq \pi_{i}(j)$ for all $j \in N$.

Kaplansky and Riordan (1946) and Touchard (1953) studied the problème des ménages, the second problem in this hierarchy. This problem is to enumerate the permutations of an $n$-set discordant with $\pi_{1}$ and $\pi_{2}$.

Riordan (1954) was the first to study the third problem in this hierarchy; 13 years later Moser (1967) also studied this problem. This problem is to enumerate the permutations of an $n$-set discordant with $\pi_{1}, \pi_{2}$ and $\pi_{3}$.

The fourth problem in this hierarchy is the enumeration of the permutations of an $n$-set discordant with $\pi_{1}, \pi_{2}, \pi_{3}$ and $\pi_{4}$. In this paper, the approach of Riordan 
$(1954,1958)$ is followed to solve this problem, the enumeration of four-discordant permutations, by means of rook polynomials and hit polynomials. If Moser's approach had been followed, then a more appropriate name for this problem would be the number of very reduced $5 \times n$ Latin rectangles. The results obtained in solving this problem support the conjecture made by Yamamoto (1956).

The rook polynomial of a rectangular board enumerates the ways nonattacking rooks can be placed on the positions of the board marked with an " $x$ ". In particular, the coefficient of $t^{k}$ in the rook polynomial expressed in the variable $t$, is the number of ways that $k$ nonattacking rooks can be placed on the marked positions of the board. The leading coefficient of the rook polynomial is the permanent of the rectangular matrix where 1 indicates the marked positions of the board and 0 indicates the unmarked positions. Minc (1964), and later Metropolis and others (1969), studied permanents associated with these problems.

The rook and hit polynomial approach is superior to the permanent approach because the coefficients of the hit polynomial yield a complete enumeration of the permutations defined on the set $N$ with respect to hits. The discordant permutations are those permutations with zero hits. The permanent approach only enumerates those permutations with zero hits.

\section{Rook polynomial recurrences}

Consider the four-ply staircase board $S_{n}$ shown in Figure 1. Note that the $S_{n}$ board has $n$ rows and $n+3$ columns. Write $S_{n}$ for its rook polynomial in the variable $t$. Based on the definition of the rook polynomial, direct computation yields $S_{n}$ for $n=1,2,3$ :

$$
\begin{aligned}
& S_{1}=1+4 t, \\
& S_{2}=1+8 t+13 t^{2}, \\
& S_{3}=1+12 t+40 t^{2}+36 t^{3} .
\end{aligned}
$$

Developing according to all positions in the first row, it is found that

$$
S_{n}=(1+t) S_{n-1}+t T_{n-1}+t U_{n-1}+t V_{n-1}
$$

where $T_{n-1}$ is the polynomial of the board (corresponding to $S_{n}$ ) with the first row and second column removed; $U_{n-1}$, with the first row and third column removed; $V_{n-1}$, with the first row and fourth column removed. 
Further development yields equations (2) and (3) which are a pair of coupled recurrences involving only $S_{i}$ and $T_{i}$.

$$
\begin{aligned}
& U_{n}=S_{n}-t S_{n-1}-\left(t+t^{2}\right) S_{n-2}+t^{3} S_{n-3}-t^{2} U_{n-2} \text { for } n \geqslant 4 . \\
& S_{n}=(1+2 t) S_{n-1}-\left(t+3 t^{2}\right) S_{n-2}+\left(t^{2}+2 t^{3}\right) S_{n-3}+t^{4} S_{n-4} \\
& \quad+t^{4} S_{n-5}-t^{6} S_{n-6}+2 t U_{n-1}+\left(t+2 t^{2}\right) U_{n-2} \text { for } n \geqslant 7 .
\end{aligned}
$$

Consider the truncated four-ply staircase board $s_{n}$ shown in Figure 1. The $s_{n}$ board can be obtained from the $S_{n}$ board by eliminating the last three columns and

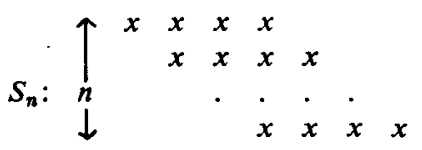

$$
\begin{aligned}
& s_{n}: \quad \begin{array}{lllll}
x & & & \\
x & x & & \\
x & x & x & \\
n & x & x & x & x
\end{array} \\
& \longleftrightarrow n+3 \longrightarrow
\end{aligned}
$$

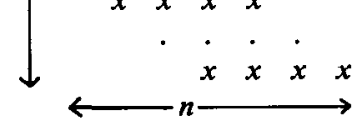

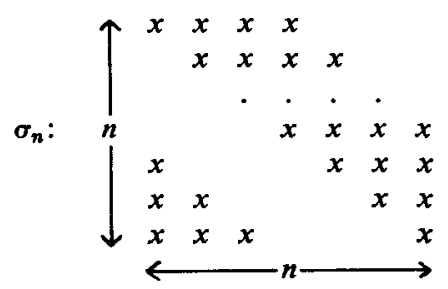$$
\begin{array}{llll}
x & x & x & \\
x & x & x & x
\end{array}
$$

FigURe 1. Rook polynomial boards.

rotating about the main diagonal. Write $s_{n}$ for its rook polynomial in the variable $t$. Direct computation yields $s_{n}$ for $n=1,2,3$ :

$$
\begin{aligned}
& s_{1}=1+t, \\
& s_{2}=1+3 t+t^{2}, \\
& s_{3}=1+6 t+7 t^{2}+t^{3} .
\end{aligned}
$$

An easy development yields the following equation:

$$
s_{n}=U_{n-1}-t S_{n-2}+t^{2} S_{n-3}+t s_{n-1} \text { for } n \geqslant 4 .
$$

Consider the completed truncation four-ply staircase board $\sigma_{n}$ shown in Figure 1. The $\sigma_{n}$ board is associated with permutations discordant with $\pi_{1}, \pi_{2}, \pi_{3}, \pi_{4}$, as defined in the introduction. The $\sigma_{n}$ board can be obtained from the $S_{n}$ board by truncating the last three columns and moving these columns to the lower left-hand corner. Write $\sigma_{n}$ for its rook polynomial in the variable $t$. Direct computation 
yields $\sigma_{n}$ for $n=1,2,3$ :

$$
\begin{aligned}
& \sigma_{1}=1+t, \\
& \sigma_{2}=1+4 t+2 t^{2}, \\
& \sigma_{3}=1+9 t+18 t^{2}+6 t^{3} .
\end{aligned}
$$

Developing according to the six positions in the lower left-hand corner yields the following equation:

$$
\sigma_{n}=s_{n}+t A_{n-1}+t B_{n-1}+t C_{n-1}+t D_{n-1}+t E_{n-1}+t F_{n-1} .
$$

Here $A_{n-1}$ is the polynomial of the board (corresponding to $\sigma_{n}$ ), with the $(n-2)$ th row and first column removed; $B_{n-1}$ is the polynomial of the $\sigma_{n}$ board, with the $(n-1)$ th row and first column removed; $C_{n-1}$ is the polynomial of the $\sigma_{n}$ board, with the $n$th row and first column removed; $D_{n-1}$ is the polynomial of the board (corresponding to $s_{n}$ ), with an additional " $x$ " placed in $(n, 3)$-position and the $(n-1)$ th row and second column removed; $E_{n-1}$ is the polynomial of the $s_{n}$ board, with the $n$th row and second column removed; $F_{n-1}$ is the polynomial of the $s_{n}$ board, with the $n$th row and third column removed.

After a long and somewhat routine development, the $A_{i}, B_{i}, C_{i}, D_{i}, E_{i}, F_{i}$ polynomials can be eliminated from equation (5); thus equation (6) is obtained.

$$
\begin{aligned}
\sigma_{n}=2 s_{n}+ & t S_{n-1}-10 t^{2} S_{n-2}+\left(t+4 t^{2}+9 t^{3}\right) S_{n-3}-\left(4 t^{3}-4 t^{4}\right) S_{n-4} \\
& +\left(5 t^{4}+t^{5}\right) S_{n-5}-\left(t^{5}+6 t^{6}\right) S_{n-6}+t^{7} S_{n-7}-(1-2 t) U_{n-1} \\
& +\left(2 t+6 t^{2}\right) U_{n-2} \text { for } n \geqslant 8 .
\end{aligned}
$$

Thus equations (2), (3), (4) and (6) form a system of recurrences that make it possible to solve for $S_{n}, U_{n}, s_{n}$ and $\sigma_{n}$ once the necessary initial values are computed from the definition of a rook polynomial. In the next section, we obtain generating functions from these recurrences.

\section{Generating functions}

Solving equations (2) and (3) simultaneously for $S_{n}$ yields the following recurrence:

$$
\begin{aligned}
& S_{n}-(1+4 t) S_{n-1}+4 t^{2} S_{n-2}+t^{4} S_{n-3}+\left(t^{2}+4 t^{3}+2 t^{4}\right) S_{n-4} \\
& -\left(3 t^{4}+4 t^{5}\right) S_{n-5}-t^{6} S_{n-7}+t^{8} S_{n-8}=0 \quad \text { for } n \geqslant 9 .
\end{aligned}
$$

A slight modification of equation (7) will yield equation (8) which is valid for $n \geqslant 0$. Let $S_{0}=1$ and $S_{i}=0$ for $i<0$. By checking equation (7) for $0 \leqslant n \leqslant 8$, equation (8) 
is obtained.

$$
\begin{aligned}
& S_{n}-(1+4 t) S_{n-1}+4 t^{2} S_{n-2}+t^{2} S_{n-3}+\left(t^{2}+4 t^{3}+2 t^{4}\right) S_{n-4} \\
& -\left(3 t^{4}+4 t^{5}\right) S_{n-5}-t^{6} S_{n-7}+t^{8} S_{n-8}=\delta_{n 0}+t^{2} \delta_{n 2} \text { for } n \geqslant 0
\end{aligned}
$$

Here $\delta_{n k}$ denotes the Kronecker delta function. From equation (8), the generating function $S(t, u)$ is expressed in terms of the two-variable polynomial $\Delta(t, u)$.

$$
\Delta(t, u) S(t, u)=1+t^{2} u^{2}
$$

where

$$
\begin{gathered}
\Delta(t, u)=1-(1+4 t) u+4 t^{2} u^{2}+t^{2} u^{3}+\left(t^{2}+4 t^{3}+2 t^{4}\right) u^{4} \\
-\left(3 t^{4}+4 t^{5}\right) u^{5}-t^{6} u^{7}+t^{8} u^{8}
\end{gathered}
$$

and

$$
S(t, u)=\sum_{n=0}^{\infty} S_{n} u^{n} .
$$

Note that equation (9) is the generating function form of equation (8).

In the same way that equation (9) was obtained, the following generating functions for $U_{n}, s_{n}$ and $\sigma_{n}$ are obtained.

$$
\begin{aligned}
& \Delta(t, u) U(t, u)=1-t u-\left(t+t^{2}\right) u^{2}+t^{3} u^{3} \\
& \begin{array}{rl}
\Delta(t, u) s(t, u)=1- & 3 t u-\left(2 t-t^{2}\right) u^{2}-\left(t+t^{2}-t^{3}\right) u^{3}+\left(3 t^{3}+3 t^{4}\right) u^{4} \\
& +\left(t^{4}-t^{5}\right) u^{5}+\left(t^{5}-t^{6}\right) u^{6}-t^{7} u^{7} \\
\Delta(t, u) \sigma(t, u)=1-3 & t u-\left(t-2 t^{2}\right) u^{2}+\left(t+5 t^{2}+2 t^{3}\right) u^{3} \\
& +\left(3 t+24 t^{2}+39 t^{3}+10 t^{4}\right) u^{4}-\left(19 t^{3}+66 t^{4}+30 t^{5}\right) u^{5} \\
& -\left(7 t^{3}+37 t^{4}+33 t^{5}\right) u^{6}-\left(3 t^{3}+27 t^{4}+64 t^{5}+30 t^{6}-4 t^{7}\right) u^{7} \\
& +\left(9 t^{5}+57 t^{6}+93 t^{7}+33 t^{8}\right) u^{8}+\left(4 t^{7}+6 t^{8}-3 t^{9}\right) u^{9} \\
& +\left(3 t^{7}+15 t^{8}+6 t^{9}-6 t^{10}\right) u^{10}-\left(3 t^{9}+15 t^{10}+10 t^{11}\right) u^{11}
\end{array}
\end{aligned}
$$

Let $P(t, u)$ denote the polynomial on the right-hand side of equation (14). The following theorem is a summary of the computation leading to equation (14).

THEOREM. The generating function for the rook polynomials of the $\sigma_{n}$ boards, is given by

$$
\sigma(t, u)=\frac{P(t, u)}{\Delta(t, u)}
$$




\section{Hit polynomials}

The hit polynomial is defined in terms of the coefficients $\sigma_{n, k}$ of the rook polynomial $\sigma_{n}$. Recalling that $\sigma_{n}$ is a polynomial in the variable $t$, the following expansion is obtained:

$$
\sigma_{n}=\sum_{k=0}^{n} \sigma_{n, k} t^{k}
$$

In equation (17), the hit polynomial is expressed in terms of the variable $y$. The coefficient of $y^{k}$ in the hit polynomial is the number of permutations of an $n$-set that have exactly $k$ hits. $A$ hit occurs when $\pi(i) \equiv i, i+1, i+2$ or $i+3(\bmod n)$ for some $i \in\{1,2, \ldots, n\}$. In particular, the coefficient of $y^{0}$ in the hit polynomial, is the number of four-discardant permutations. The hit polynomial, see Riordan (1954, 1958), is obtained from equation (16) by replacing $t^{k}$ with $(n-k) !(y-1)^{k}$.

$$
N_{n}(y)=\sum_{k=0}^{n} \sigma_{n, k}(n-k) !(y-1)^{k}=\sum_{k=0}^{n} N_{n, k} y^{k} .
$$

From equations (10) and (14), the following equation is derived.

$$
\begin{aligned}
\sigma_{n}=(1+4 t) & \sigma_{n-1}-4 t^{2} \sigma_{n-2}-t^{2} \sigma_{n-3}-\left(t^{2}+4 t^{3}+2 t^{4}\right) \sigma_{n-4} \\
& +\left(3 t^{4}+4 t^{5}\right) \sigma_{n-5}+t^{6} \sigma_{n-7}-t^{8} \sigma_{n-8} \text { for } n \geqslant 12 .
\end{aligned}
$$

Using the coefficient notation introduced in equation (16), the following equation is derived from equation (18):

$$
\begin{gathered}
\sigma_{n, k}=\sigma_{n-1, k}+4 \sigma_{n-1, k-1}-4 \sigma_{n-2, k-2}-\sigma_{n-3, k-2}-\sigma_{n-4, k-2} \\
-4 \sigma_{n-4, k-3}-2 \sigma_{n-4, k-4}+3 \sigma_{n-5, k-4}+4 \sigma_{n-5, k-5}+\sigma_{n-7, k-6} \\
-\sigma_{n-8, k-8} \text { for } n \geqslant 12 .
\end{gathered}
$$

Using equation (19) to replace $\sigma_{n, k}$ in equation (17) and doing a considerable amount of simplification, the following equation is obtained.

$$
\begin{aligned}
& N_{n}(y)=[4 y+n-4] N_{n-1}(y)-[y-1] N_{n-1}^{\prime}(y)-4[y-1]^{2} N_{n-2}(y) \\
&-(n-2)[y-1]^{2} N_{n-3}(y)+[y-1]^{3} N_{n-3}^{\prime}(y) \\
&-(y-1)^{2}\left[2(y-1)^{2}+4(n-3)(y-1)+(n-2)(n-3)\right] N_{n-4}(y) \\
&+2(2 y+n-5)[y-1]^{3} N_{n-4}^{\prime}(y)-[y-1]^{4} N_{n-4}^{\prime}(y) \\
&+(4 y+3 n-16)[y-1]^{4} N_{n-5}(y)-3[y-1]^{5} N_{n-5}^{\prime}(y) \\
&+(n-6)[y-1]^{6} N_{n-7}(y)-[y-1]^{7} N_{n-7}^{\prime}(y)-[y-1]^{8} N_{n-8}(y)
\end{aligned}
$$

for $n \geqslant 12$. 
Here the prime denotes differentiation with respect to the variable $y$. Table 1 gives the coefficients $\sigma_{n, k}$ for $0 \leqslant n \leqslant 11$. Table 2 gives the coefficients $N_{n, k}$ for $4 \leqslant n \leqslant 11$. The $N_{n, k}$ coefficients have combinatorial meaning only for $n \geqslant 4$. Equation (20) can be used to compute the polynomials $N_{n}(y)$ for $n \geqslant 12$.

TABLE 1

Coefficients $\sigma_{n, k}$

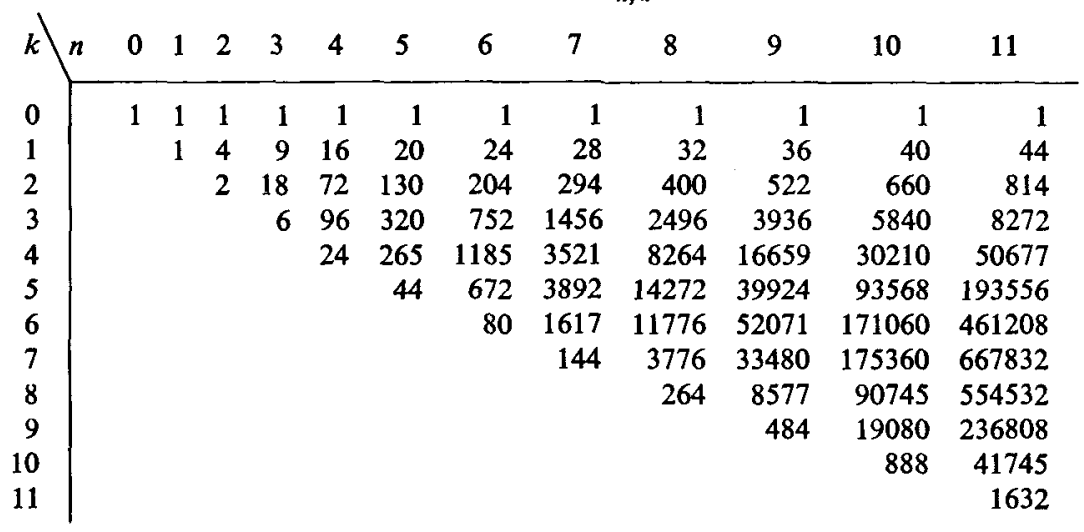

TABLE 2

Coefficients $N_{n, k}$

\begin{tabular}{|c|c|c|c|c|c|c|c|c|}
\hline \multirow{2}{*}{$k \backslash$} & \\
\hline & 4 & 5 & 6 & 7 & 8 & 9 & 10 & 11 \\
\hline 0 & 0 & 1 & 2 & 31 & 264 & 2783 & 30818 & 369321 \\
\hline 1 & 0 & 0 & 24 & 154 & 1664 & 15984 & 173000 & 2004486 \\
\hline 2 & 0 & 10 & 60 & 595 & 4512 & 44802 & 457040 & 5159517 \\
\hline 3 & 0 & 20 & 152 & 980 & 8832 & 74784 & 759920 & 8282472 \\
\hline 4 & 24 & 45 & 210 & 1421 & 9776 & 90396 & 853100 & 9261714 \\
\hline 5 & & 44 & 192 & 1106 & 8832 & 70452 & 707744 & 7464996 \\
\hline 6 & & & 80 & 609 & 4512 & 43206 & 405800 & 4539282 \\
\hline 7 & & & & 144 & 1664 & 15768 & 180560 & 1989768 \\
\hline 8 & & & & & 264 & 4221 & 49730 & 673893 \\
\hline 9 & & & & & & 484 & 10200 & 145926 \\
\hline 10 & & & & & & & 888 & 23793 \\
\hline 11 & & & & & & & & 1632 \\
\hline
\end{tabular}

\section{A tribonacci sequence}

Yamamoto (1956) observed that the Lucas numbers (which form a Fibonacci sequence) are found as the leading coefficients of the rook polynomials associated with the three-discordant permutations. Thus it is reasonable to find a 'tribonacci' sequence as the leading coefficients of the $\sigma_{n}$ polynomials. Observe the diagonal entries in Table 1. 
THEOREM. The leading coefficients of the $\sigma_{n}$ polynomials satisfy the following tribonacci recurrence:

$$
\sigma_{n, n}=\sigma_{n-1, n-1}+\sigma_{n-2, n-2}+\sigma_{n-3, n-3}-4 \text { for } n \geqslant 7
$$

Proof. By studying the contribution to the leading coefficient of $\sigma_{n}$, denoted $l\left(\sigma_{n}\right)$, by the terms on the right-hand side of equation (6), the following equation is obtained:

$$
\begin{aligned}
\sigma_{n, n}=l\left(\sigma_{n}\right)=2 l\left(S_{n}\right) & +l\left(S_{n-1}\right)-10 l\left(S_{n-2}\right)+9 l\left(S_{n-3}\right)+4 l\left(S_{n-4}\right) \\
& +l\left(S_{n-5}\right)-6 l\left(S_{n-6}\right)+l\left(S_{n-7}\right)+2 l\left(U_{n-1}\right)+6 l\left(U_{n-2}\right)
\end{aligned}
$$

for $n \geqslant 8$.

Since the $s_{n}$ board is triangular for all $n$, it follows that $l\left(s_{n}\right)=1$. Applying equation (22) to $\sigma_{i, i}$ for $i=n, n-1, n-2$ and $n-3$, the following equation is derived:

$$
\begin{aligned}
\sigma_{n, n}- & \sigma_{n-1, n-1}-\sigma_{n-2, n-2}-\sigma_{n-3, n-3} \\
= & -4+l\left(S_{n-1}\right)-11 l\left(S_{n-2}\right)+18 l\left(S_{n-3}\right)+4 l\left(S_{n-4}\right)-2 l\left(S_{n-5}\right)-20 l\left(S_{n-6}\right) \\
& +2 l\left(S_{n-7}\right)+4 l\left(S_{n-8}\right)+5 l\left(S_{n-9}\right)-l\left(S_{n-10}\right)+2 l\left(U_{n-1}\right) \\
& +4 l\left(U_{n-2}\right)-8 l\left(U_{n-3}\right)-8 l\left(U_{n-4}\right)-6 l\left(U_{n-5}\right) \text { for } n \geqslant 11 .
\end{aligned}
$$

From equations (2) and (3), equations (24) and (25) are obtained by studying the contribution of the leading coefficients $l\left(U_{i}\right)$ and $l\left(S_{i}\right)$.

$$
\begin{gathered}
l\left(U_{i}\right)=l\left(S_{i}\right)-l\left(S_{i-1}\right)-l\left(S_{i-2}\right)+l\left(S_{i-3}\right)-l\left(U_{i-2}\right) \text { for } i \geqslant 4 . \\
l\left(S_{i}\right)=2 l\left(S_{i-1}\right)-3 l\left(S_{i-2}\right)+2 l\left(S_{i-3}\right)+l\left(S_{i-4}\right)-l\left(S_{i-6}\right) \\
+2 l\left(U_{i-1}\right)+2 l\left(U_{i-2}\right) \quad \text { for } i \geqslant 7 .
\end{gathered}
$$

By applying equations (24) and (25) for $i=n-1, n-2, n-3$ and $n-4$ to equation (23), the following equation is obtained.

$$
\sigma_{n, n}-\sigma_{n-1, n-1}-\sigma_{n-2, n-2}-\sigma_{n-3, n-3}=-4 \text { for } n \geqslant 11 .
$$

For $n=7,8,9$ and 10, direct computation with the values given in Table 1 completes the proof. 


\section{Acknowledgements}

The author thanks John Riordan for his helpful suggestions and encouragement. Part of this research was done while the author was a visitor at the University of Queensland; this visit was made possible by a Travel Grant awarded by the University of Queensland. The computer computations for this research were done on the University of Pittsburgh's DEC-10 system.

\section{References}

G. Berman and K. D. Fryer (1972), Introduction to combinatorics (Academic Press, New York), $70-72$.

I. Kaplansky and J. Riordan (1946), 'The problème des ménages', Scripta Math. 12, 113-124.

N. Metropolis, M. L. Stein and P.R. Stein (1969), 'Permanents of cyclic $(0,1)$ matrices', J. Combinatorial Theory 7, 291-321.

H. Minc (1964). 'Permanents of $(0,1)$ circulants', Canad. Math. Bull. 7, 253-263.

W. O. J. Moser (1967), 'The number of very reduced $4 \times n$ Latin rectangles', Canad. J. Math. 19, $1011-1017$.

J. Riordan (1954), 'Discordant permutations', Scripta Math. 20, 14-23.

J. Riordan (1958), Introduction to combinatorial analysis (Wiley, New York), 178-180.

J. Touchard (1953), 'Permutations discordant with two given permutations', Scripta Math. 19, 109-119.

K. Yamamoto (1956), 'Structure polynomial of Latin rectangles and its application to a combinatorial problem', Mem. Fac. Sci. Kyusyu Univ. 10 (Series A), 1-13.

Department of Mathematics and Statistics

University of Pittsburgh

Pittsburgh, PA 15260

U.S.A. 